\title{
ATRA protects skin fibroblasts against UV-induced oxidative damage through inhibition of E3 ligase Hrd1
}

\author{
XIANYE CHENG $^{1 *}$, WEN QIAN ${ }^{1 *},{\text { FANG } \text { CHEN }^{2} \text {, YI JIN }}^{1}$, FENGDI WANG $^{1}$, \\ XIAOYI LU ${ }^{1}$, SAE ROM LEE ${ }^{1}$, DONGMING SU ${ }^{3}$ and BIN CHEN ${ }^{1}$ \\ ${ }^{1}$ Department of Dermatology and Venereology, The First Affiliated Hospital of Nanjing Medical University; \\ ${ }^{2}$ Department of Biochemistry and Molecular Biology, Nanjing Medical University; \\ ${ }^{3}$ Center of Metabolic Disease Research, Nanjing Medical University, Nanjing, Jiangsu 210029, P.R. China
}

Received November 22, 2018; Accepted May 29, 2019

DOI: $10.3892 / \mathrm{mmr} .2019 .10450$

\begin{abstract}
All-trans retinoic acid (ATRA) can protect fibroblasts against ultraviolet (UV)-induced oxidative damage, however, its underlying molecular mechanism is still unclear. The present study aimed to investigate the role of 3-hydroxy-3-methylglutaryl reductase degradation (Hrd1) in the protective effect of ATRA on human skin fibroblasts exposed to UV. The expression of Hrd1 in human or mice skin was assessed by immunohistochemistry (IHC) staining and western blot analysis. Hrd1 siRNA (si-Hrd1) and Hrd1 recombinant adenoviruses (Ad-Hrd1) were used to downregulate and upregulate Hrd1 expression in fibroblasts, respectively. The interaction between Hrd1 and NF-E2-related factor 2 (Nrf2) was assessed by co-immunoprecipitation (co-IP) and immunofluorescence analysis. The results revealed that Hrd1 expression was increased but $\mathrm{Nrf} 2$ expression was decreased in UV-exposed human skin fibroblasts. In addition, ATRA could reverse the increase of Hrd1 expression induced by UV radiation in vivo and in vitro. ATRA or knockdown of Hrd1 could increase Nrf2 expression in fibroblasts exposed to UV radiation, and $\mathrm{Hrd} 1$ could directly interact with $\mathrm{Nrf} 2$ in skin fibroblasts. Notably, overexpression of Hrd1 abolished the protective effect of ATRA on the UV-induced decrease of Nrf2 expression, the production of reactive oxygen species (ROS) and the decrease of cell viability. In conclusion, the present data demonstrated that ATRA protected skin fibroblasts against UV-induced oxidative damage through inhibition of E3 ligase Hrd1.
\end{abstract}

Correspondence to: Professor Bin Chen,Department of Dermatology and Venereology, The First Affiliated Hospital of Nanjing Medical University, 300 Guangzhou Road, Nanjing, Jiangsu 210029, P.R. China E-mail: chenbin@njmu.edu.cn

${ }^{*}$ Contributed equally

Key words: oxidative damage, ultraviolet, Hrd1, Nrf2, ATRA

\section{Introduction}

Chronic ultraviolet (UV) exposure is harmful and hazardous to the human skin. Excessive UV light irradiation can penetrate the skin and impair cellular antioxidant defense in dermal human fibroblasts (HDFs), which play an important role in maintaining normal structure and function of the skin $(1,2)$. Consequently, high production of reactive oxygen species (ROS) leads to cellular oxidative stress, cell damage and apoptosis $(3,4)$. Since the generation of ROS has been associated with the pathogenesis of UV-mediated damage in skin cells, many antioxidants have been used to protect HDFs against UV through induction of NF-E2-related factor 2(Nrf2)-dependent antioxidant gene expression $(5,6)$.

The transcription factor Nrf2 could bind antioxidant response elements (AREs) and regulate the expression of antioxidant genes such as NQO1, $\gamma$-GCS, HO-1 and others (7). It has been reported that $\mathrm{Nrf} 2$ acts as a master regulator of the cellular antioxidant defense against cutaneous photodamage mediated by UV radiation $(8,9)$. Notably, Nrf2 knock-out mice displayed UV-induced sunburn reaction and oxidative DNA damage compared to wild-type mice (10). As a result, pharmacological activation of $\mathrm{Nrf} 2$ has been a novel approach to skin photoprotection. All-trans retinoic acid (ATRA) has been clinically used to treat several skin diseases including UV-induced skin oxidative damage $(11,12)$. Tan et al reported that ATRA could activate Nrf2 and induce Nrf2 target genes expression (13). However, whether ATRA could increase Nrf2 expression to protect skin fibroblasts against UV-induced oxidative damage remains unclear.

3-Hydroxy-3-methylglutaryl reductase degradation (Hrd1), also known as synoviolin, is a endoplasmic reticulum-associated degradation (ERAD)-associated E3 ubiquitin ligase (14). It contains a RING finger domain at the $\mathrm{C}$-terminus required for ubiquitin ligase activity. HRD1 is implicated in the pathogenesis of rheumatoid arthritis, Alzheimer's disease, liver fibrosis, renal fibrosis, Parkinson's disease, cancer and Wolfram syndrome (15-19). Wu et al (16) demonstrated that Hrd1 is the specific E3 ubiquitin ligase of Nrf2 and induces Nrf2 ubiquitylation and degradation in embryonic fibroblast cells. Although Hrd1 could be upregulated in response to oxidative stress, little is known about the role of Hrd1 in UV-induced 
skin oxidative damage. Using skin tissues, upregulation of Hrd1 expression and downregulation of Nrf2 expression were detected in UV-exposed fibroblasts from human skin. It was also revealed that ATRA could reverse the increase of Hrd1 expression induced by UV radiation in vivo and in vitro. The aim of the present study was to investigate the role of Hrd1 in the protective effect of ATRA on human skin fibroblasts exposed to UV.

\section{Materials and methods}

Collection of clinical skin samples. Samples of human skin $(n=10)$ were obtained from surgeries at the First Affiliated Hospital of Nanjing Medical University. Samples were divided into 2 groups with the same sex (all female) and age (average age of sun-exposed group: $40.20 \pm 11.39$; average age of sun-protected group: $40.00 \pm 10.32$ ). The sun-protected group consisted of breast or back skin areas and the sun-exposed group consisted of face or neck skin areas. For immunohistochemical analysis, the skin biopsies were placed in $10 \%$ phosphate-buffered formalin. The present study was approved by the Local Ethics Committees of the First Affiliated Hospital of Nanjing Medical University (Nanjing, China) (Permit no. 2016-SRFA-033). Written informed consent was obtained from all participants in this study.

Animal model. BALB/ C mice (age, 6-8 weeks; weight, 18-22 g; 20 male and 20 female) were obtained from the Chinese Academy of Sciences, Shanghai SLAC Laboratory Animal Co., Ltd., and maintained in a pathogen-free barrier facility at Nanjing Medical University. The mice were maintained at $22-23^{\circ} \mathrm{C}, 55-60 \%$ humidity and a 12 -h light/dark cycle, and provided with certified standard chow and tap water ad libitum. Mice were randomly divided into 4 groups: the control group, the UV group, the ATRA group and the UV+ATRA group. The dorsal area of all mice was shaved with electric clippers twice a week. The UV source was supplied by Bio-spectra system (Vilber Lourmat) with a UVB fluorescent bulb with a peak wavelength at $312 \mathrm{~nm}$ and a UVA fluorescent bulb with a peak wavelength at $365 \mathrm{~nm}$. Mice in the ATRA group received 0.1\% ATRA cream (15 mg ATRA:15 g cream; Chongqing Huapont Pharmaceutical Co., Ltd.) on the back, coating $0.1 \mathrm{ml}$ every time. Mice in the UV+ATRA group received ATRA cream, and then were treated with UV. Mice were exposed to UVA $10 \mathrm{~J} / \mathrm{cm}^{2}$, UVB $30 \mathrm{~mJ} / \mathrm{cm}^{2}$ every day for 14 weeks and the duration of the exposure was $1 \mathrm{~h} /$ day. During the entire experimental process, all efforts were made to minimize the suffering of the animals, in accordance with the Institutional Animal Care and Use Committee (IACUC) of Nanjing Medical University.

Immunohistochemistry (IHC) and immunofluorescence analyses. Human and mice skin tissues were prepared for immunohistochemical analysis. Sections (6- $\mu \mathrm{m}$ thick) were immunolabeled with primary antibodies (Hrd1 1:150; cat. no. ab118483; Nrf2 1:200; cat. no. ab62352; Abcam). For immunofluorescent staining, fibroblasts were incubated with primary antibodies (Hrd1, 1:50; Nrf2, 1:100) for $1 \mathrm{~h}$ at room temperature. After washing with PBST, cells were incubated with secondary antibodies [Alexa546- (1:200; cat. no. A10040;
Thermo Fisher Scientific, Inc,) or Alexa488- (1:200; cat. no. A21206; Thermo Fisher Scientific, Inc.) conjugated donkey anti-rabbit IgG, Alexa350- (1:200; cat. no. A21081; Thermo Fisher Scientific, Inc.) or Alexa546- (1:200; cat. no. A11056; Thermo Fisher Scientific, Inc.) conjugated donkey anti-goat Ig) for $45 \mathrm{~min}$. Images were captured by LSM 700 confocal laser scanning microscope (Zeiss AG), equipped with a ZEN 2009 software for image acquisition and analyses.

Cell culture and treatment. HDFs were aseptically isolated from healthy adult male circumcised foreskins with the approval of the Local Ethics Committees of the First Affiliated Hospital of Nanjing Medical University (Permit no. 2016-SRFA-033). Written informed consent was obtained from all subjects in this study. Normal skin samples were sterilized in $70 \%$ ethanol, minced, put in collagenase for 1 hour and incubated in Dulbecco's modified Eagle's medium (DMEM, Gibco; Thermo Fisher Scientific, Inc.) supplemented with $10 \%$ fetal bovine serum (FBS; Sigma-Aldrich; Merck KGaA) and 1\% penicillin-streptomycin (Beijing Solarbio Science \& Technology Co., Ltd.) in an atmosphere of $5 \% \mathrm{CO}_{2}$ at $37^{\circ} \mathrm{C}$. HDFs were washed once with PBS and then exposed to UVA irradiation $\left(10 \mathrm{~J} / \mathrm{cm}^{2}\right)$ or UVB irradiation $\left(30 \mathrm{~mJ} / \mathrm{cm}^{2}\right)$. ATRA (product no. R2625; Sigma-Aldrich; Merck KGaA) was used to treat fibroblasts according to a previous study (20). The dosage of ATRA was $1 \mu \mathrm{mol} / 1$.

Real-time RT-PCR. Total RNA was extracted from cell samples by using TRIzol (Invitrogen; Thermo Fisher Scientific, Inc.). RNA samples were used to synthesize cDNA through RT using SuperScript III Reverse Transcriptase kit (Thermo Fisher Scientific, Inc.) according to manufacturer's instructions. PCR reaction systems were prepared using $\mathrm{SYBR}^{\circledR}$-Green Quantitative RTqPCR kit (Sigma-Aldrich; Merck KGaA). Primers used to identify Hrd1 were: Forward, 5'-AACCCCTGGGACAACA AGG-3' and reverse, 5'-TCTGAGCTAGGGATGCTGGT-3'; and the primers for NRF2 were: Forward, 5'-ACACGGTCC ACAGCTCATC-3' and reverse, 5'-TGTCAATCAAATCCA TGTCCTG-3'. GAPDH was used as an internal control: Forward, 5'-TGTTGCCATCAATGACCCCTT-3' and reverse, 5'-CTCCACGACGTACTCAGCG-3'. Real-time RT-PCR was carried out using the following thermocycling conditions: $95^{\circ} \mathrm{C}$ for $50 \mathrm{sec}$, followed by 40 cycles at $95^{\circ} \mathrm{C}$ for $12 \mathrm{sec}$ and $60^{\circ} \mathrm{C}$ for $45 \mathrm{sec}$. All data were processed using the $2^{-\Delta \Delta \mathrm{Cq}}$ method (21).

Western blot analysis. Cells were washed twice in ice-cold PBS, and then solubilized in RIPA lysis buffer (Vazyme). Proteins extracted were quantified using a Bicinchoninic Acid Protein Assay kit (cat. no. P0012; Beyotime Institute of Biotechnology). Equal amounts of each protein sample (30 $\mu \mathrm{g}$ /lane) were subjected to $10 \%$ SDS-PAGE and were transferred to polyvinylidene fluoride membranes, which were blocked with 5\% skimmed milk at room temperature for $1 \mathrm{~h}$. Members were incubated overnight at $4{ }^{\circ} \mathrm{C}$ with antibodies targeting Hrd1 (cat. no. ab170901; 1:2,000; Abcam), Nrf2 (cat. no. sc-13032; 1:2,000; Santa Cruz Biotechnology, Inc.) and $\beta$-actin (cat.no. 58169, 1:1,000; Cell Signaling Technology, Inc.), followed by incubation with a horseradish peroxidase-labeled goat anti-rabbit secondary antibody (cat. no. ab6721; 1:3,000; Abcam) for $1.5 \mathrm{~h}$ at room temperature. $\beta$-Actin was used as a 
A
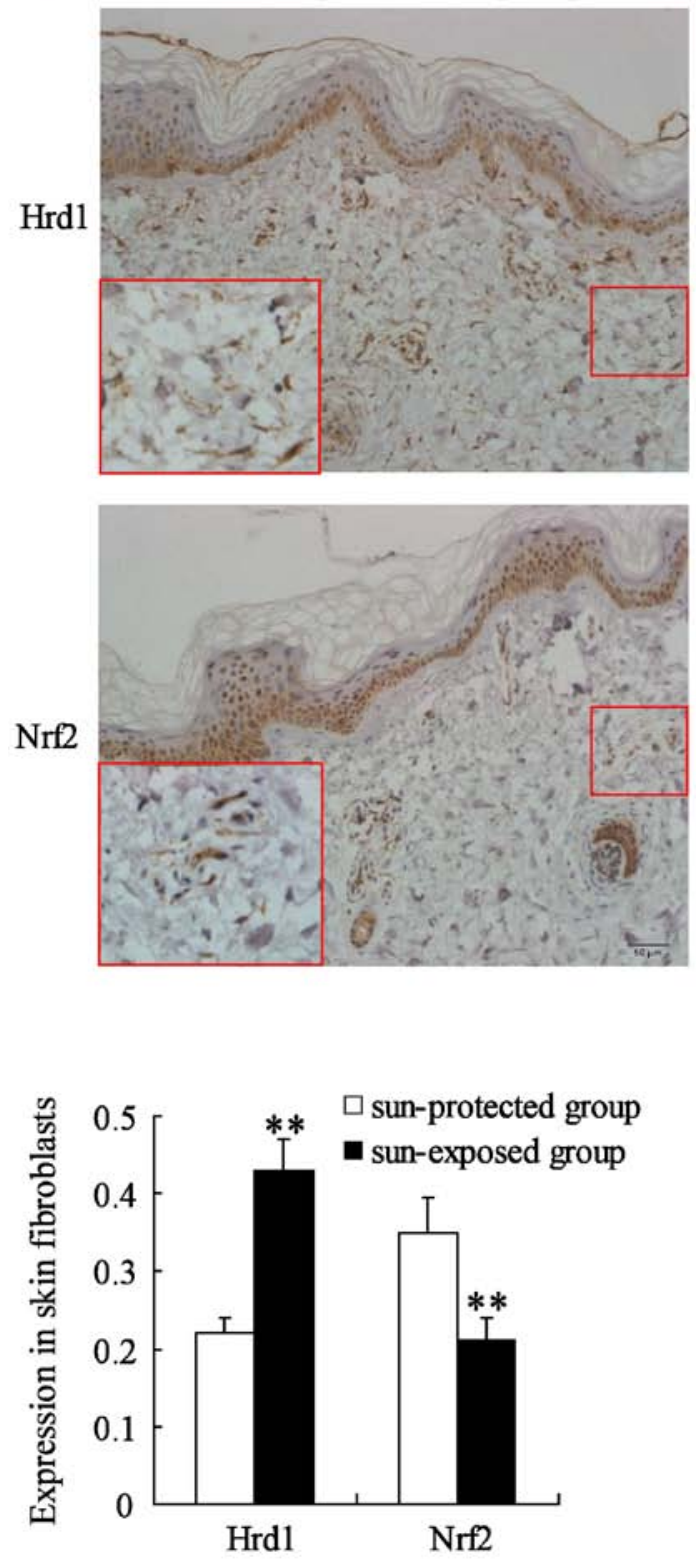

sun-exposed group
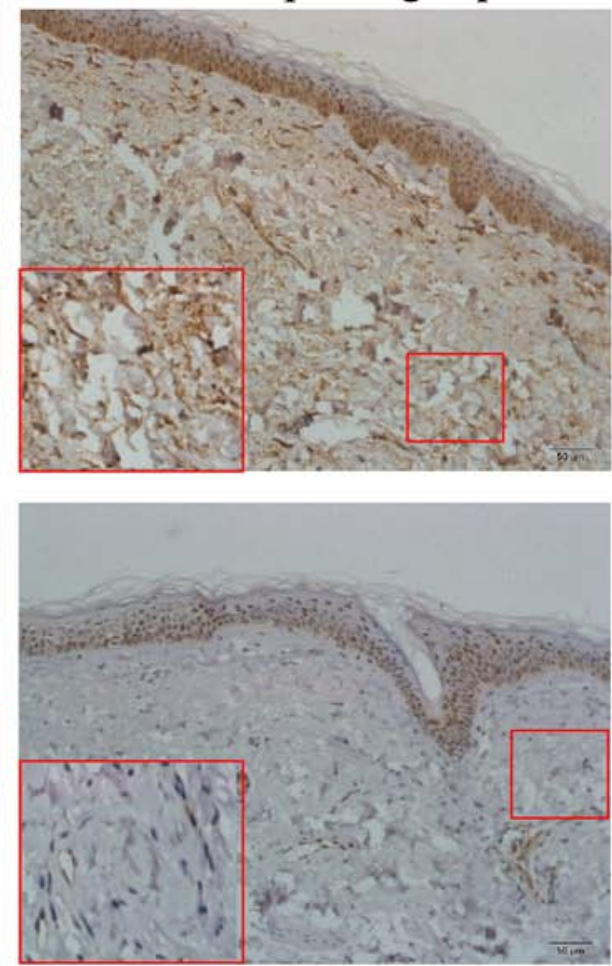

B

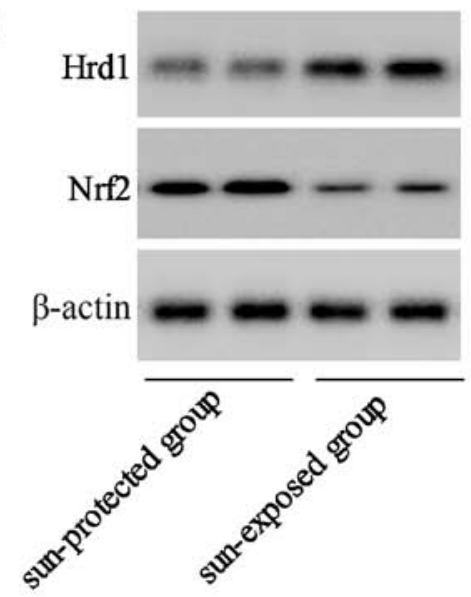

Figure 1. UV-induced upregulation of Hrd1 expression and downregulation of Nrf2 expression in human skin. Hrd1 and Nrf2 expression in fibroblasts from human skin were measured using (A) immunohistochemical staining of Hrd1 or Nrf2 (magnification, x200 and inset x400; n=10) and (B) western blotting. ${ }^{* * *} \mathrm{P}<0.01$, compared to the sun-protected group. UV, ultraviolet; Hrd1, 3-hydroxy-3-methylglutaryl reductase degradation; Nrf2, NF-E2-related factor 2.

loading control. Protein bands were visualized by enhanced chemiluminescence (cat. no. WBKLS0500; EMD Millipore). ImageJ 1.45 software (National Institutes of Health) was used to perform densitometric analysis of each band.

Co-immunoprecipitation (Co-IP). The lysates of fibroblasts were incubated with anti-Hrd1 antibody, anti-Nrf2 antibody, or control IgG for $1 \mathrm{~h}$, followed by incubation overnight with protein $\mathrm{A} / \mathrm{G}$ agarose beads (Thermo Fisher Scientific, Inc.). The beads were collected by centrifugation $\left(14,000 \mathrm{x} \mathrm{g}\right.$ at $4^{\circ} \mathrm{C}$ for $1 \mathrm{~min}$ ), washed three times with the lysis buffer and resuspended in $1 \mathrm{X}$ SDS loading buffer. The IP were eluted from the beads by incubation at $95^{\circ} \mathrm{C}$ for $5 \mathrm{~min}$. The eluted proteins were separated by $10 \%$ SDS-PAGE and western blotting was subsequently performed with the indicated antibodies.
Small interfering RNA and recombinant adenoviruses infection. Small interfering RNA specific for Hrd1 (si-Hrd1-1 and si-Hrd1-2) and control siRNA (si-control) were synthesized (Guangzhou RiboBio Co., Ltd.) and transfected using Lipofectamine 2000. The sequences of si-Hrd1-1 and si-Hrd1-2 were: 5'-CCAUGAGGCAGUUCAAGAAdTdT-3' and 5'-UGU CUGGCCUUCACCGUUU-3, respectively. Human fibroblasts ( $2 \times 10^{5} /$ well) were infected with $1 \times 10^{8}$ pfu of Hrd1 recombinant adenoviruses according to the manufacturer's instructions (GeneChem, Shanghai, China). The expression level of Hrd1 was assessed by western blotting.

Intracellular reactive oxygen species (ROS) detection. Intracellular ROS content were assessed by determination of 2', 7'-dichlorofluorescin diacetate (DCFH-DA, Beyotime Institute 
A

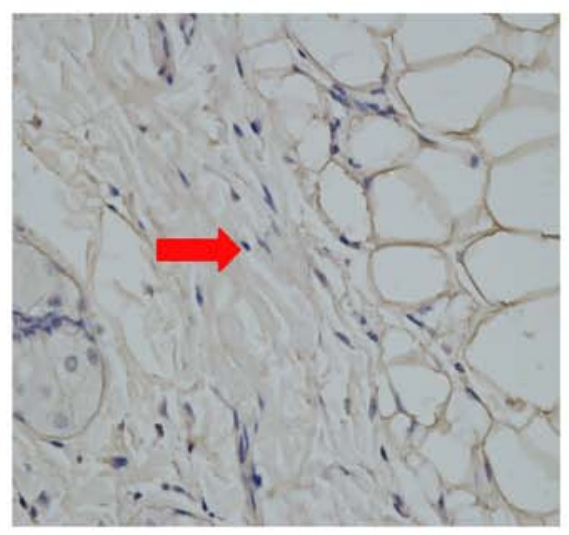

ATRA

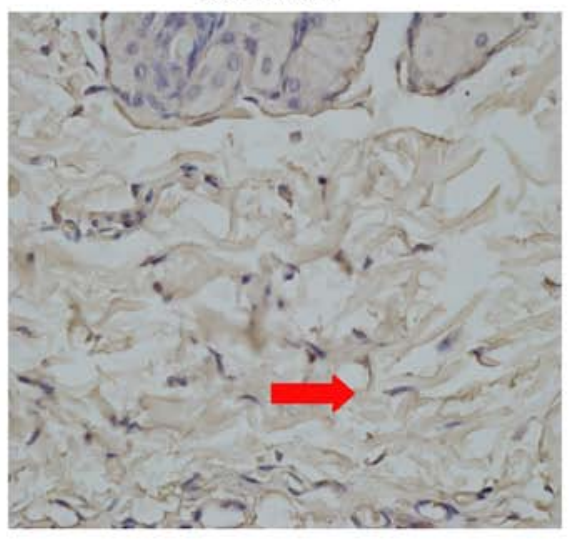

B

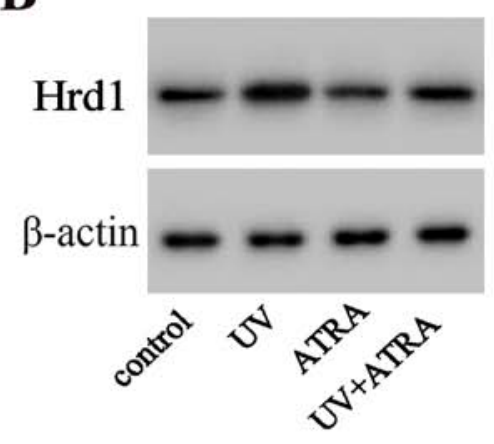

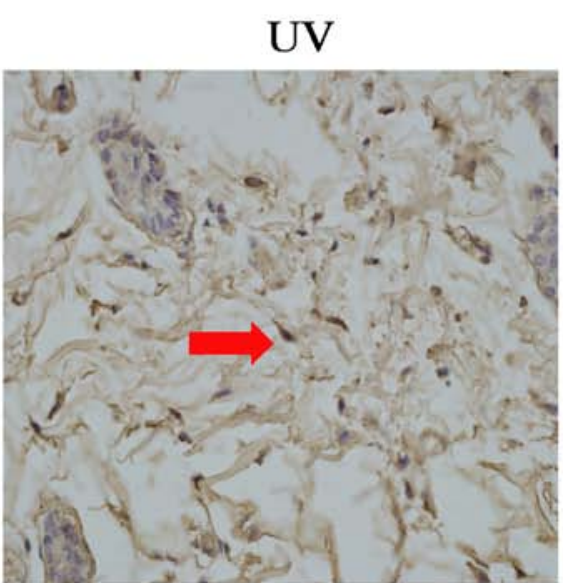

UV+ATRA
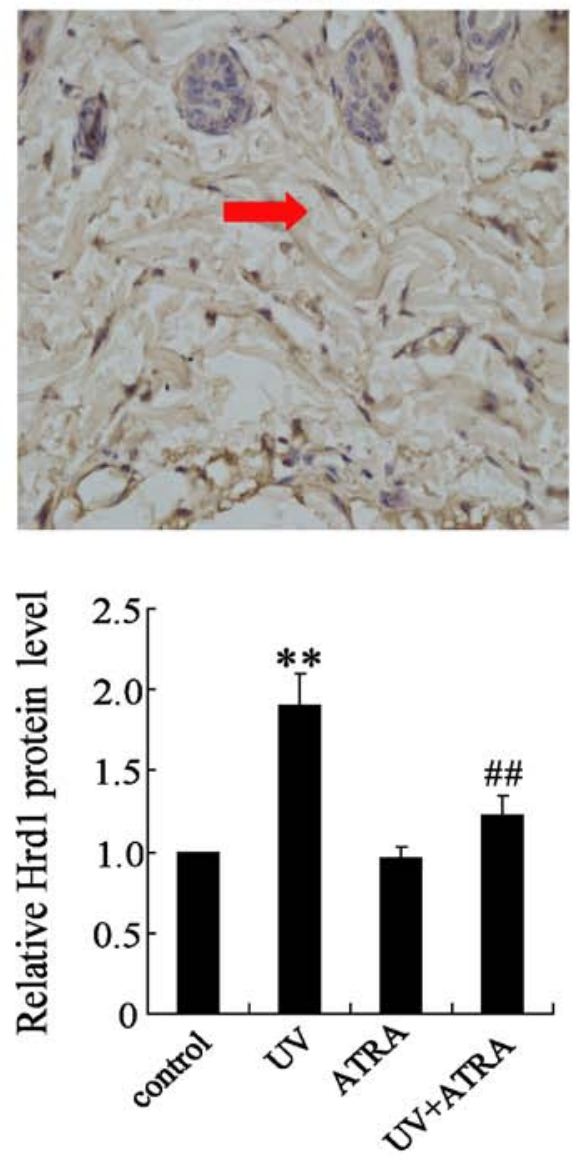

Figure 2. ATRA reverses Hrd1 expression increased by UV radiation in an animal model. Hrd1 expression in skin fibroblasts from mice skin using (A) immunohistochemical staining of Hrd1 (magnification $\mathrm{x} 400 ; \mathrm{n}=10$ ) and $(\mathrm{B})$ western blotting. Skin fibroblasts are indicated by red arrows. ${ }^{* *} \mathrm{P}<0.01$, compared to the control; ${ }^{\# \#} \mathrm{P}<0.01$, compared to UV. ATRA, all-trans retinoic acid; Hrd1, 3-hydroxy-3-methylglutaryl reductase degradation; UV, ultraviolet.

of Biotechnology, Inc.), which is converted into fluorescent 2', 7'-dichlorofluorescin (DCF) in the presence of peroxides (22). Human fibroblasts were incubated with a $10-\mu \mathrm{M}$ DCFH-DA probe for $30 \mathrm{~min}$ and then harvested and washed with PBS. Images were captured using a fluorescence microscope (DP70; Olympus Corporation) and the fluorescence (emission, $525 \mathrm{~nm}$; excitation, $488 \mathrm{~nm}$ ) was assessed using a fluorescence plate reader (BD Falcon; BD Biosciences).

WST-1 assay. Cell viability was determined using the WST-1 assay. Human fibroblasts were seeded in 48-well dishes
(4X10 4 cells/well) in $200 \mu \mathrm{l}$ culture medium and transfected and treated as aforementioned for $48 \mathrm{~h}$. Then, each well was supplemented with $20 \mu \mathrm{l}$ WST-1 (Roche Diagnostics) and incubated for $3 \mathrm{~h}$. The absorbance of the samples was measured with a spectrophotometer reader.

Statistical analysis. The data in the present study were analyzed using SPSS 19.0 (IBM Corporation) statistical software and presented as the means \pm SD. The analysis to determine the statistical differences among the groups was performed using the Student's t-test or one-way analysis of variance (ANOVA) 

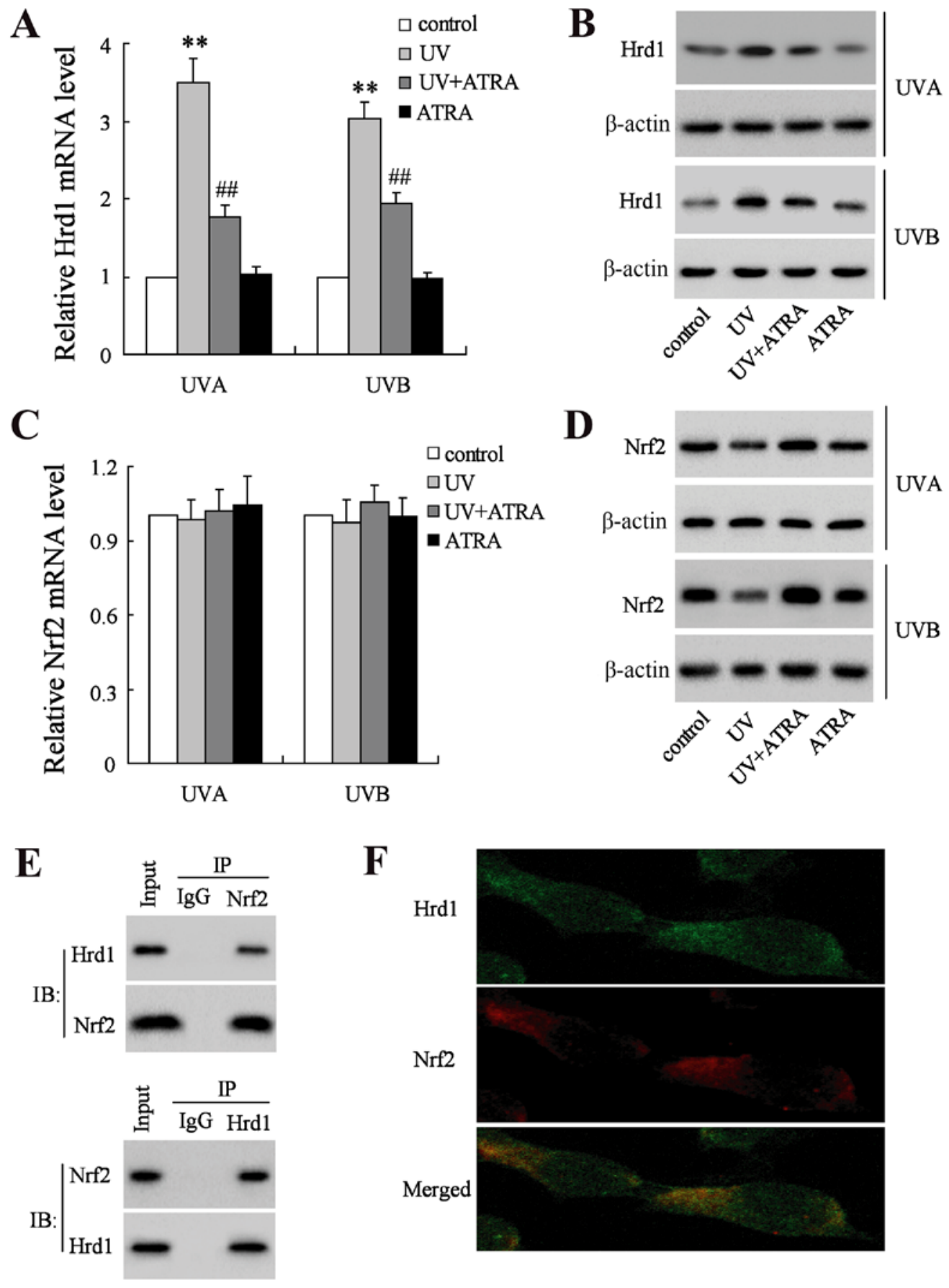

Figure 3. ATRA decreases Hrd1 expression and increases Nrf2 expression in human fibroblasts exposed to UV radiation. Human fibroblasts were exposed to UVA and UVB, and then treated with ATRA for $24 \mathrm{~h}$. (A) The mRNA level of Hrd1 was assessed using real-time RT-PCR and (B) the protein level of Hrd1 was assessed using western blotting. (C) The mRNA level of Nrf2 was assessed using real-time RT-PCR and (D) the protein level of Nrf2 was assessed using western blotting. (E) Fibroblasts were pretreated with MG132 $(10 \mu \mathrm{M})$ for $6 \mathrm{~h}$ and the endogenous protein-protein interactions between Hrd1 and Nrf2 were assessed by IP with Hrd1 antibody (upper image) or Nrf2 antibody (lower image), followed by IB with Hrd1 antibody and Nrf2 antibody. IgG was used as a negative control for IP. (F) Representative images of Hrd1 interaction with Nrf2. Fibroblasts were stained with Hrd1 (green) and Nrf2 (red). Hrd1 and Nrf2 merged appeared as orange/yellow. Compared to control, ${ }^{* *} \mathrm{P}<0.01$; compared to UV; ${ }^{\# \#} \mathrm{P}<0.01$. ATRA, all-trans retinoic acid; Hrd1, 3-hydroxy-3-methylglutaryl reductase degradation; Nrf2, NF-E2-related factor 2; UV, ultraviolet; IP, immunoprecipitation; IB, immunoblotting.

followed by Student-Newman-Keuls post hoc test. A P-value $<0.05$ was considered to indicate a statistically significant difference.

\section{Results}

UV induces upregulation of Hrdl expression and downregulation of Nrf2 expression in human skin. The expression of
Hrd1 and Nrf2 was first evaluated in human skin fibroblasts from a sun-protected group (10 samples) and a sun-exposed group (10 samples) with same sex and age. IHC and western blot analysis revealed that the expression level of Hrd1 was significantly increased but the expression level of Nrf2 was significantly decreased in fibroblasts from sun-exposed skin compared to that in fibroblasts from sun-protected skin $(\mathrm{P}<0.01)$ (Fig. 1). 


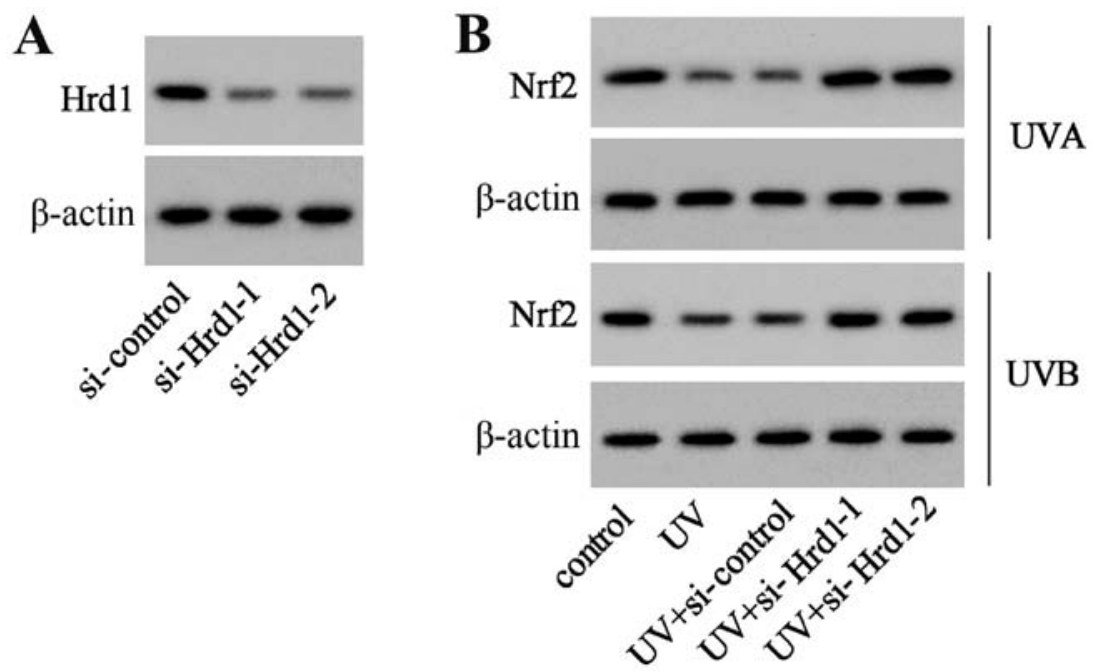

Figure 4. Downregulation of Hrd1 expression increases Nrf2 expression in UV-irradiated human dermal fibroblasts. (A) Human fibroblasts were transfected with si-Hrd1-1 and si-Hrd1-2 for $48 \mathrm{~h}$, and then, the protein level of Hrd1 was assessed. (B) Human fibroblasts were transfected with si-Hrd1-1 and si-Hrd1-2 for $24 \mathrm{~h}$, and then, exposed to UVA and UVB. The protein level of Nrf2 was assessed. Hrd1, 3-hydroxy-3-methylglutaryl reductase degradation; Nrf2, NF-E2-related factor 2; UV, ultraviolet.
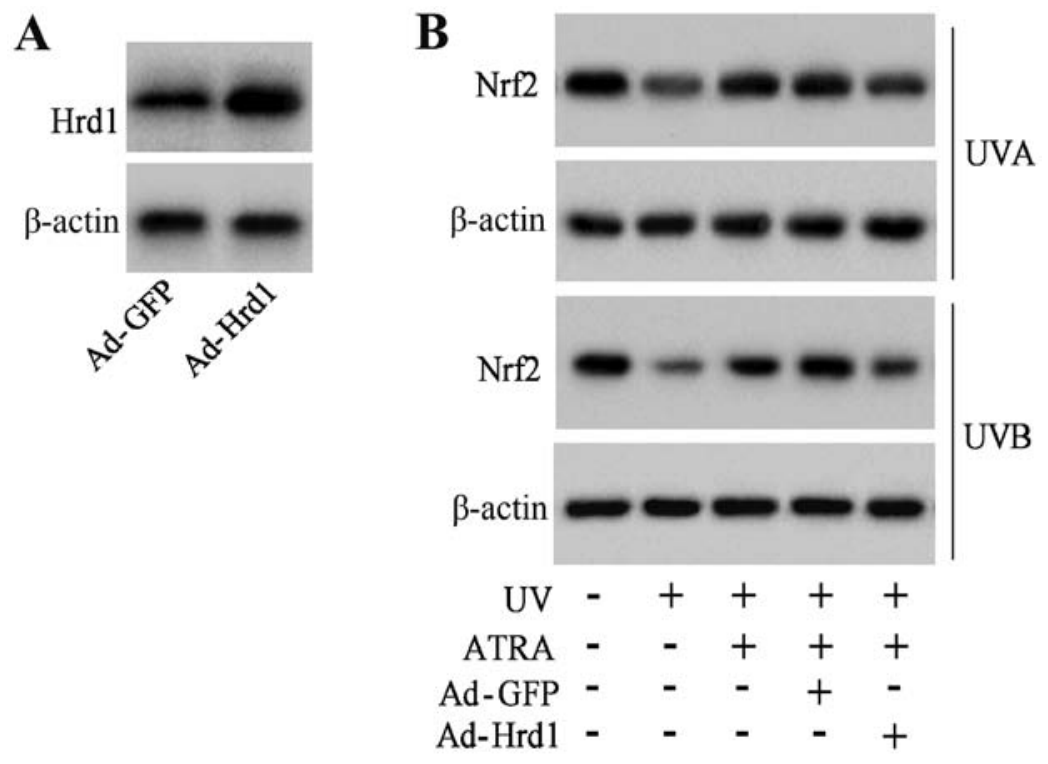

Figure 5. Overexpression of Hrd1 abolishes the protective effect of ATRA against the UV-induced decrease of Nrf2 expression. (A) Human fibroblasts were infected with Ad-Hrd1 for $48 \mathrm{~h}$, and then, the protein level of Hrd1 was assessed. (B) Human fibroblasts were infected with Ad-Hrd1 for $24 \mathrm{~h}$. Then, cells were exposed to UVA and UVB and treated with ATRA for another $24 \mathrm{~h}$. The protein level of Nrf2 was assessed. Hrd1, 3-hydroxy-3-methylglutaryl reductase degradation; ATRA, all-trans retinoic acid; UV, ultraviolet; Nrf2, NF-E2-related factor 2.

ATRA reverses $H r d l$ expression increased by $U V$ radiation in an animal model. The effect of ATRA on Hrd1 expression was also evaluated in fibroblasts from mice exposed to UV radiation. IHC and western blot analysis revealed that expression level of Hrd1 was significantly increased in fibroblasts from mice exposed to UV compared with the control group, which could be partially reversed by ATRA (Fig. 2).

ATRA decreases Hrdl expression and increases Nrf2 expression in human fibroblasts exposed to $U V$ radiation. UVA and UVB increased the mRNA and protein levels of Hrd1, which could be reversed by ATRA in human fibroblasts (Fig. 3A and B). Next, the mRNA and protein level of Nrf2 was assessed. As revealed in Fig. 3C, neither UV nor
ATRA had an effect on the mRNA level of Nrf2. Notably, the level of Nrf2 protein was significantly decreased in human fibroblasts exposed UV radiation, which could be restored by ATRA (Fig. 3D). To explore whether Hrd1 could interact with Nrf2 in fibroblasts, Co-IP and immunofluorescence analyses were used to assess the co-localization between Hrd1 and Nrf2. Co-IP analysis demonstrated that endogenously expressed $\mathrm{Hrd} 1$ and $\mathrm{Nrf} 2$ co-existed in precipitated complexes in fibroblasts (Fig. 3E). In addition, Hrd1 co-localized with the Nrf2 in fibroblasts as revealed in Fig. 3F.

Downregulation of $\mathrm{Hrdl}$ expression increases the expression level of Nrf 2 in UV-irradiated human dermal fibroblasts. To investigate whether Hrd1 was involved in the decrease of Nrf2 
A

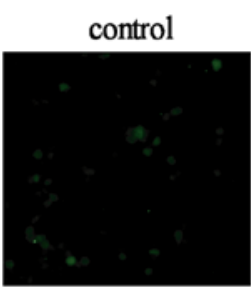

control

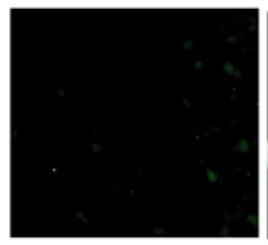

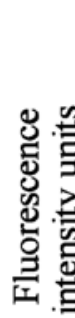

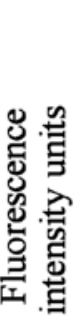

140

120

I*

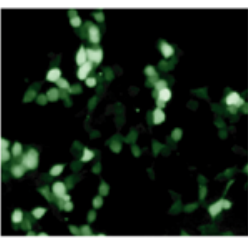

UVB+ATRA
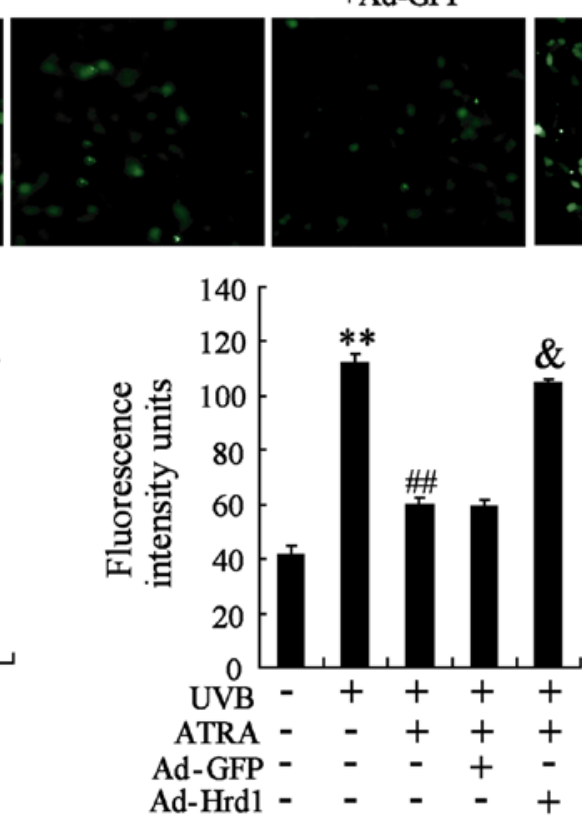

B

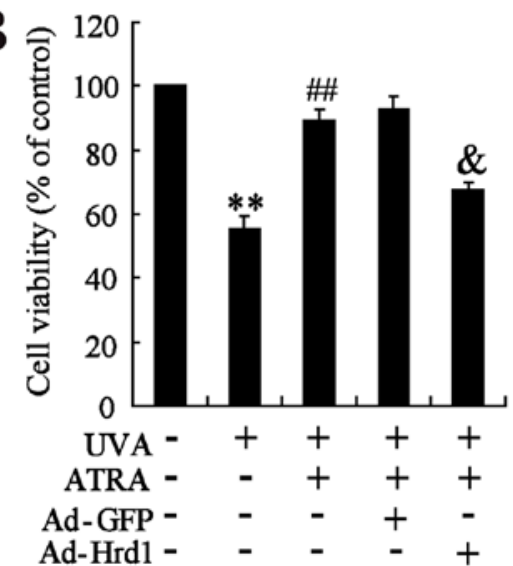

+Ad-GFP

Ad-Hrd1 - - - - +

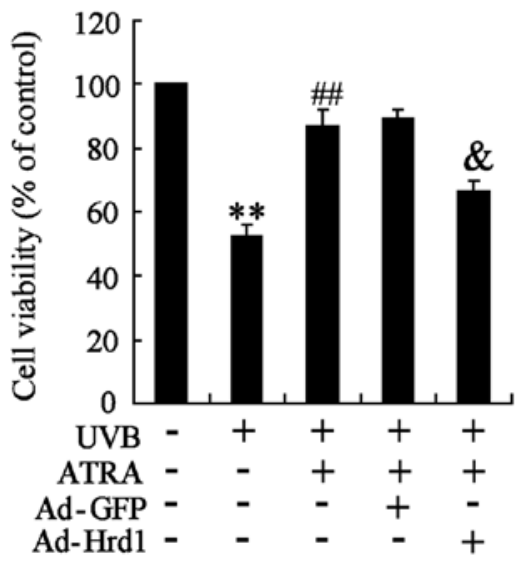

UVA+ATRA

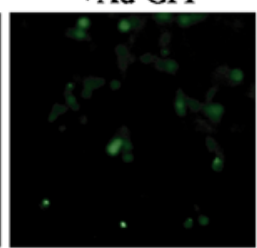

UVB+ATRA

+Ad-GFP

UVB+ATRA

+ Ad-Hrd 1

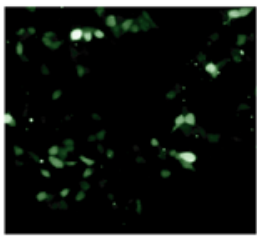

Figure 6. Overexpression of Hrd1 abolishes the protective effect of ATRA against UV-induced ROS production and cytotoxicity. Human fibroblasts were infected with ad-Hrd1 for $24 \mathrm{~h}$, and then, exposed to UVA and UVB, and treated with ATRA for $24 \mathrm{~h}$. (A) Fluorescence analyses were performed using a fluorescence microscope and quantified ROS production was detected. (B) Cell viability was assessed by WST-1 assay. Compared to control, ${ }^{* *} \mathrm{P}<0.01$; compared to UV, ${ }^{\# /} \mathrm{P}<0.01$; compared to UV+ATRA+Ad-GFP, ${ }^{\circledR} \mathrm{P}<0.01$. Hrd1, 3-hydroxy-3-methylglutaryl reductase degradation; ATRA, all-trans retinoic acid; UV, ultraviolet; ROS, reactive oxygen species.

expression induced by UV, siRNA was used to knockdown Hrd1 expression in human dermal fibroblasts. As revealed in Fig. 4A, Hrd1 siRNA (si-Hrd1-1 and si-Hrd1-2) could decrease Hrd1 expression, indicating that si-Hrd1-1 and si-Hrd1-2 were efficiently introduced into human fibroblasts and acted to knock down Hrd1. After UV exposure, the expression of Nrf2 was significantly decreased. Silencing of Hrd1 could increase Nrf2 expression in human fibroblasts exposed to $\mathrm{UV}$ radiation (Fig. 4B).
Overexpression of Hrdl abolishes the protective effect of ATRA against the UV-induced decrease of Nrf2 expression. To investigate that ATRA prevented the decrease of Nrf2 protein expression against UV radiation through Hrd1, human fibroblasts were infected with Hrd1 recombinant adenoviruses (Ad-Hrd1). As revealed in Fig. 5A, Ad-Hrd1 significantly increased Hrd1 expression. ATRA treatment significantly increased Nrf2 expression in fibroblasts exposed to UV, which was reversed by overexpression of Hrd1 (Fig. 5B). 
Overexpression of Hrdl abolishes the protective effect of ATRA against $U V$-induced ROS production and cytotoxicity. The role of Hrd1 on the protective effect of ATRA against UV-induced oxidative stress was assessed in human dermal fibroblasts. As revealed in Fig. 6A, ATRA treatment efficiently inhibited UV-induced ROS production, which could be abolished by overexpression of Hrd1. Next, the role of Hrd1 on the protective effect of ATRA against UV-induced cytotoxicity was investigated. As observed in Fig. 6B, ATRA treatment significantly increased viability of fibroblasts exposed to UV radiation. Similarly, overexpression of Hrd1 abolished the protective effect of ATRA against UV-induced cytotoxicity.

\section{Discussion}

ATRA has been clinically used to treat several skin diseases including UV-induced skin oxidative damage $(11,12)$. In the present study, it was demonstrated that Hrd1 was involved in the protective effect of ATRA against UV-induced ROS production and cytotoxicity in skin fibroblasts. Hrd1 expression was significantly increased in UV-exposed human or mice skin fibroblasts. Moreover, ATRA could reverse the increase of Hrd1 expression induced by UV radiation in vivo and in vitro. In addition, ATRA or knockdown of Hrd1 could increase Nrf2 expression in fibroblasts exposed to UV radiation, and Hrd1 could directly interact with Nrf2 in skin fibroblasts. Notably, overexpression of Hrd1 abolished the protective effect of ATRA on the UV-induced decrease of Nrf2 expression, the production of reactive oxygen species (ROS) and the decrease of cell viability.

Hrd1 has been demonstrated to be upregulated in response to oxidative stress (23). In the present study, it was determined that the level of Hrd1 was higher in UV-exposed human or mice skin fibroblasts. A previous study revealed that Hrd1 was the specific E3 ubiquitin ligase of Nrf2 for ubiquitylation and degradation $(16,24)$. It was revealed that Hrd1 and Nrf2 were both in the cytoplasm, and interaction between Hrd1 and Nrf2 was strong in skin fibroblasts. It was also determined that there was an inverse correlation of HRD1 expression and Nrf2 expression in fibroblasts treated with UV radiation. Notably, knockdown of Hrd1 could reverse the decrease of Nrf2 expression in fibroblasts exposed to UV radiation. In the present study, a connection among UV radiation, Hrd1 increase, and Nrf2 degradation was established. Hrd1 was responsible for the decrease of Nrf2 under UV light irradiation.

It has been reported that Nrf2 acts as a master regulator of the cellular antioxidant defense against cutaneous photodamage mediated by UV radiation $(8,9)$. However, the effect of UV irradiation on Nrf2 expression and activity in skin fibroblasts has remained controversial. Certain studies demonstrated that UV irradiation led to Nrf2 activation and accumulation in the nucleus $(25,26)$, while others revealed that $\mathrm{Nrf} 2$ was downregulated and transported from the nucleus in dermal fibroblasts exposed to UV irradiation $(27,28)$. The different results may be due to the cell source, the time and the dose of UV exposure in different laboratories. It was observed that UV could downregulate Nrf2 expression using human-derived fibroblasts. Given that Nrf2 could combat oxidative damage, it was speculated that pharmacological upregulation of Nrf2 could protect skin fibroblasts against UV-induced injury.
ATRA has been reported to protect skin fibroblasts against UV-induced injury during which oxidative stress plays an important pathological role $(29,30)$. The role of ROS and antioxidant signaling was therefore examined in the ATRA-mediated protective effect. It was demonstrated that ATRA could abolish the decrease of Nrf2 expression in fibroblasts exposed to UV radiation. Although ATRA at high concentrations $(\geq 30 \mu \mathrm{mol} / \mathrm{l})$ can induce cytotoxicity and activate Nrf2 (13), low concentrations of ATRA alone $(1 \mu \mathrm{mol} / \mathrm{l})$ had no effect on Nrf2 expression (Fig. 3D) and cell viability (data not shown) in the present study. Thus, ATRA treatment only resulted in the decrease of ROS production induced by UV. These results led us to ask the question of how ATRA eliminated UV-induced ROS generation. The present study, for the first time, revealed that the ubiquitin E3 ligate Hrd1 was involved in the protective effect of ATRA on UV-induced oxidative damage in skin fibroblasts.

In conclusion, our observations revealed that ATRA decreased Hrdl expression in skin fibroblasts exposed to UV irradiation, leading to upregulation of Nrf2 expression and ultimately to the reduction of ROS production and cytotoxicity. The present study shed light on the molecular mechanisms responsible for the protective effect of ATRA on skin fibroblasts against UV-induced oxidative damage.

\section{Acknowledgements}

Not applicable.

\section{Funding}

The present study was supported by the National Natural Science Foundation of China Grant (no. 81673082) and the Six Talent Peaks Project of Jiangsu Province (no. 2008101).

\section{Availability of data and materials}

All data generated and/or analyzed during the present study are available from the corresponding author upon reasonable request.

\section{Authors' contributions}

$\mathrm{XC}$ and WQ performed the experiments, analyzed the data and drafted the manuscript. FC performed the supplementary experiments, contributed to the interpretation of the data and revised the manuscript. YJ and SRL performed the experiments of the clinical skin samples and the animal model. FW and XL assisted with the molecular biology experiments. DS and $\mathrm{BC}$ conceived and designed the study and revised the manuscript. All authors read and approved the final manuscript and agree to be accountable for all aspects of the research in ensuring that the accuracy or integrity of any part of the work are appropriately investigated and resolved.

\section{Ethics approval and consent to participate}

This study was approved by the Local Ethics Committees of the First Affiliated Hospital of Nanjing Medical University (Nanjing, China) (Permit no. 2016-SRFA-033). Written 
informed consent was obtained from all participants in this study. All animal protocols were approved by the Institutional Animal Care and Use Committee (IACUC) of Nanjing Medical University.

\section{Patient consent for publication}

Not applicable.

\section{Competing interests}

The authors declare that they have no competing interests.

\section{References}

1. Vedrenne N, Coulomb B, Danigo A, Bonté F and Desmoulière A The complex dialogue between (myo)fibroblasts and the extracellular matrix during skin repair processes and ageing. Pathol Biol (Paris) 60: 20-27, 2012.

2. Cavinato $M$ and Jansen-Dürr P: Molecular mechanisms of UVB-induced senescence of dermal fibroblasts and its relevance for photoaging of the human skin. Exp Gerontol 94: 78-82, 2017.

3. Wenk J, Brenneisen P, Meewes C, Wlaschek M, Peters T, Blaudschun R, Ma W, Kuhr L, Schneider L and ScharffetterKochanek K: UV-induced oxidative stress and photoaging. Curr Probl Dermatol 29: 83-94, 2001.

4. Oliveira MM, Daré RG, Barizão ÉO, Visentainer JV, Romagnolo MB, Nakamura CV and Truiti MDCT: Photodamage attenuating potential of Nectandra hihua against UVB-induced oxidative stress in L929 fibroblasts. J Photochem Photobiol B 181: 127-133, 2018.

5. Liang B, Peng L, Li R, Li H, Mo Z, Dai X, Jiang N, Liu Q, Zhang E, Deng $\mathrm{H}$, et al: Lycium barbarum polysaccharide protects HSF cells against ultraviolet-induced damage through the activation of Nrf2. Cell Mol Biol Lett 23: 18, 2018.

6. Parzonko A and Kiss AK: Caffeic acid derivatives isolated from Galinsoga parviflora herb protected human dermalfibroblasts from UVA-radiation. Phytomedicine 57: 215-222, 2019.

7. Silva-Palacios A, Ostolga-Chavarría M, Zazueta $C$ and Königsberg M: Nrf2: Molecular and epigenetic regulation during aging. Ageing Res Rev 47: 31-40, 2018.

8. Zhong JL, Edwards GP, Raval C, Li H and Tyrrell RM: The role of Nrf 2 in ultraviolet a mediated heme oxygenase 1 induction in human skin fibroblasts. Photochem Photobiol Sci 9: 18-24, 2010.

9. Schäfer M, Dütsch S, auf dem Keller U and Werner S: Nrf2: A central regulator of UV protection in the epidermis. Cell Cycle 9: 2917-2918, 2010

10. Saw CL, Huang MT, Liu Y, Khor TO, Conney AH and Kong AN: Impact of Nrf2 on UVB-induced skin inflammation/photoprotection and photoprotective effect of sulforaphane. Mol Carcinog 50: 479-486, 2011.

11. Francz PI, Conrad J and Biesalski HK: Modulation of UVA-induced lipid peroxidation and suppression of UVB-induced ornithine decarboxylase response by all-trans retinoic acid in human skin fibroblasts in vitro. Biol Chem 379: 1263-1269, 1998.

12. Hiraishi Y, Hirobe S, Iioka H, Quan YS, Kamiyama F, Asada H, Okada N and Nakagawa S: Development of a novel therapeutic approach using a retinoic acid-loaded microneedle patch for seborrheic keratosis treatment and safety study in humans. J Control Release 171: 93-103, 2013

13. Tan KP, Kosuge K, Yang M and Ito S: NRF2 as a determinant of cellular resistance in retinoic acid cytotoxicity. Free Radic Biol Med 45: 1663-1673, 2008.

14. Kaneko M, Okuma Y and Nomura Y: Molecular approaches to the treatment, prophylaxis, and diagnosis of Alzheimer's disease: Possible involvement of HRD1, a novel molecule related to endoplasmic reticulum stress, in Alzheimer's disease. J Pharmacol Sci 118: 325-330, 2012.
15. Amano T, Yamasaki S, Yagishita N, Tsuchimochi K, Shin H, Kawahara K, Aratani S, Fujita H, Zhang L, Ikeda R, et al: Synoviolin/Hrd1, an E3 ubiquitin ligase, as a novel pathogenic factor for arthropathy. Genes Dev 17: 2436-2449, 2003.

16. Wu T, Zhao F, Gao B, Tan C, Yagishita N, Nakajima T, Wong PK, Chapman E, Fang D and Zhang DD: Hrd1 suppresses Nrf2-mediated cellular protection during liver cirrhosis. Genes Dev 28: 708-722, 2014.

17. Kim H, Bhattacharya A and Qi L: Endoplasmic reticulum quality control in cancer: Friend or foe. Semin Cancer Biol 33: 25-33, 2015.

18. Nomura J, Hosoi T, Kaneko M, Ozawa K, Nishi A and Nomura Y Neuroprotection by endoplasmic reticulum stress-induced HRD1 and chaperones: Possible therapeutic targets for Alzheimer's and Parkinson's disease. Med Sci (Basel) 4: pii E14, 2016.

19. Fonseca SG, Ishigaki S, Oslowski CM, Lu S, Lipson KL, Ghosh R, Hayashi E, Ishihara H, Oka Y, Permutt MA and Urano F: Wolfram syndrome 1 gene negatively regulates ER stress signaling in rodent and human cells. J Clin Invest 120: 744-755, 2010.

20. Shim JH, Shin DW, Noh MS and Lee TR: Reduced collagen internalization via down-regulation of MRC 2 expression by UVA irradiation and its recovery by all-trans retinoic acid. J Dermatol Sci 73: 163-166, 2014.

21. Livak KJ and Schmittgen TD: Analysis of relative gene expression data using real-time quantitative PCR and the 2(-Delta Delta C(T)) method. Methods 25: 402-408, 2001.

22. Afri M, Frimer AA and Cohen Y: Active oxygen chemistry within the liposomal bilayer. Part IV: Locating 2',7'-dichlorofluorescein (DCF), 2',7'-dichlorodihydrofluorescein (DCFH) and 2',7'-dichlorodihydrofluorescein diacetate (DCFH-DA) in the lipid bilayer. Chem Phys Lipids 131: 123-133, 2004.

23. Saito R, Kaneko M, Kitamura Y, Takata K, Kawada K, Okuma Y and Nomura Y: Effects of oxidative stress on the solubility of HRD1, a ubiquitin ligase implicated in Alzheimer's disease. PLoS One 9: e94576, 2014.

24. O'Connell MA and Hayes JD: The Keap1/Nrf2 pathway in health and disease: From the bench to the clinic. Biochem Soc Trans 43: 687-689, 2015.

25. Hirota A, Kawachi Y, Itoh K, Nakamura Y, Xu X, Banno T, Takahashi T, Yamamoto $M$ and Otsuka F: Ultraviolet A irradiation induces NF-E2-related factor 2 activation in dermal fibroblasts: Protectiverole in UVA-induced apoptosis. J Invest Dermatol 124: 825-832, 2005.

26. Gegotek A, Rybałtowska-Kawałko P and Skrzydlewska E: Rutin as a mediator of lipid metabolism and cellular signaling pathways interactions in fibroblasts altered by UVA and UVB radiation. Oxid Med Cell Longev 2017: 4721352, 2017.

27. Saito Y, Tsuruma K, Ichihara K, Shimazawa M and Hara H: Brazilian green propolis water extract up-regulates the early expression level of HO-1 and accelerates Nrf2 after UVA irradiation. BMC Complement Altern Med 15: 421, 2015.

28. Kannan S and Jaiswal AK: Low and high dose UVB regulation of transcription factor NF-E2-related factor 2. Cancer Res 66: 8421-8429, 2006.

29. Nilsson J, Gritli-Linde A and Heby O: Skin fibroblasts from spermine synthase-deficient hemizygous gyro male $(\mathrm{Gy} / \mathrm{Y})$ mice overproduce spermidine and exhibit increased resistance to oxidative stress but decreased resistance to UV irradiation. Biochem J 352: 381-387, 2000.

30. Wu PY, Huang CC, Chu Y, Huang YH, Lin P, Liu YH, Wen KC, Lin CY, Hsu MC and Chiang HM: Alleviation of ultraviolet B-induced photodamage by coffea arabica extract in human skin fibroblasts and hairless mouse skin. Int J Mol Sci 18: E782, 2017.

This work is licensed under a Creative Commons Attribution-NonCommercial-NoDerivatives 4.0 International (CC BY-NC-ND 4.0) License. 